\title{
VALORES DE REFERÊNCIA DE QUALIDADE PARA METAIS PESADOS EM SOLOS NO ESTADO DO ESPÍRITO SANTO ${ }^{(1)}$
}

\author{
Henrique de Sá Paye ${ }^{(2)}$, Jaime Wilson Vargas de Mello ${ }^{(3)}$, Walter Antônio \\ Pereira Abrahão ${ }^{(3)}$, Elpídio Inácio Fernandes Filho ${ }^{(3)}$, Lívia Cristina Pinto \\ $\operatorname{Dias}^{(4)}$, Maria Luisa Oliveira Castro ${ }^{(4)}$, Stefeson Bezerra de Melo ${ }^{(5)} \&$ Michele \\ Milanez França ${ }^{(2)}$
}

\section{RESUMO}

Valores de referência de qualidade (VRQ) para metais pesados em solos, em âmbito mundial, são bastante heterogêneos. No Brasil, poucos estudos para estabelecer esses valores foram concluídos. Na ausência de VRQ para metais pesados em solos, estabelecidos para as condições do Estado do Espírito Santo, utilizam-se valores genéricos internacionais ou desenvolvidos para outros Estados. Entretanto, deve-se ressaltar que o uso desses valores pode levar a avaliações inadequadas, já que existem diferenças nas condições técnicas e variáveis ambientais de cada região, em especial das condições geológicas, hídricas e pedológicas. Essas diferenças justificam o desenvolvimento de uma tabela própria com VRQ para metais pesados em solos, adequada às condições do Estado do Espírito Santo. Nesse sentido, o presente estudo buscou obter os teores naturais de 10 metais pesados e verificar a distribuição desses elementos nos solos das bacias hidrográficas Riacho, Reis Magos e Santa Maria da Vitória, no Estado do Espírito Santo, tendo em vista o estabelecimento de Valores de Referência de Qualidade (VRQ). Para isso, foram determinados os teores totais por ataque ácido, com digestão em forno micro-ondas, conforme o método SW-846 3052 da Environmental Protection Agency (USEPA) e dosagem por espectrofotometria de emissão óptica com plasma induzido (ICP- OES). Os teores totais extraídos nos solos dessas bacias hidrográficas são relativamente baixos e inferiores aos reportados para solos de regiões brasileiras e de outros países. Os teores de cádmio

\footnotetext{
(1) Parte da Tese de Mestrado do primeiro autor apresentada à Universidade Federal de Viçosa - UFV. Recebido para publicação em setembro de 2009 e aprovado em agosto de 2010.

${ }^{(2)}$ Doutorando do Programa de Pós-Graduação em Solos e Nutrição de Plantas da Universidade Federal de Viçosa - UFV. CEP 36571- 000 Viçosa (MG). Bolsista da CAPES. E-mails: henriquepaye@yahoo.com.br; michellegeoufv@yahoo.com.br

(3) Professor do Departamento de Solos, UFV. E-mail: jwvmello@ufv.br; wabrahao@ufv.br; elpidio@ufv.br

(4) Estudante de graduação em Engenharia Ambiental, UFV. E-mails: liviacrisdias@yahoo.com.br; maria_luizacastro@hotmail.com

(5) Engenheiro-Agrônomo, Departamento de Ciências Ambientais, Universidade Federal Rural do Semi-Árido UFERSA.CEP 59625-900 Mossoró (RN). E-mail: stefeson@hotmail.com
} 
ficaram abaixo do limite de detecção (LD) do método para todas as amostras. Em consequência, os VRQ obtidos para 12 metais pesados no Estado do Espírito Santo foram semelhantes ou inferiores aos obtidos para outras regiões do Brasil e do mundo. Os VRQ obtidos a partir do percentil 75 (quartil superior) para As $(<12,83)$, Cd $(<0,13)$, Co $(10,21), \mathrm{Cr}(54,13), \mathrm{Cu}(5,91)$, Mn $(137,80)$, Mo $(1,74), \mathrm{Ni}(9,17), \mathrm{Pb}$ $(<4,54), \mathrm{V}(109,96)$ e $\mathrm{Zn}(29,87)$, em mg kg-1, encontram-se próximos ou abaixo daqueles obtidos para o Estado de São Paulo e para solos brasileiros, de modo geral.

Termos de indexação: elementos potencialmente tóxicos, micronutrientes, teores naturais de metais pesados em solos.

\section{SUMMARY: REFERENCE QUALITY VALUES FOR HEAVY METALS IN SOILS FROM ESPÍRITO SANTO STATE, BRAZIL}

The Reference Quality Values (RQV) for heavy metals in soils are quite heterogeneous around the world. In Brazil, few studies establishing such values have been published. In the absence of VRQ of heavy metals in soils established for the specific conditions of the State of Espirito Santo, international generic values or values developed for other States are used. However, it should be noted that the use of these values can lead to erroneous assessments, due to the regional differences in technical and environmental variables, particularly in terms of geological, water and soil conditions. The purpose of this study was to determine heavy metal concentration in soils of Espirito Santo State, and to investigate the major factors involved in the distribution of $\mathrm{As}, \mathrm{Cd}, \mathrm{Co}, \mathrm{Cr}, \mathrm{Cu}, \mathrm{Mn}, \mathrm{Mo}, \mathrm{Ni}, \mathrm{Pb}, \mathrm{Ti}, \mathrm{V}$ and $\mathrm{Zn}$ in soils of the river basins of Riacho, Reis Magos and Santa Maria da Vitória. Based on this information RQV were established. Total concentrations were determined by microwave oven digestion, according to the SW-846 3052 method of the Environmental Protection Agency (USEPA), and by Inductively Coupled Plasma Optical Emission Spectrometer (ICP-OES). The mean concentrations of most heavy metals studied were, in general, lower than reported in international references. Cadmium concentrations were below the detection limit $(D L)$ in all samples. The RQVs found from the percentile 75 for As (<12.83), Cd (<0.13), Co (10.21), Cr (54.13), Cu (5.91), $\mathrm{Mn}$ (137.80), Mo (1.74), Ni (9.17), Pb (<4.54), V(109.96), and Zn (29.87), in $\mathrm{mg} \mathrm{kg}^{-1}$, were similar to or lower than those adopted in Sao Paulo state and in Brazilian, in general.

Index terms: potentially toxic elements, micronutrients, natural concentration of heavy metals in soil.

\section{INTRODUÇÃO}

A distribuição de metais pesados nos solos, sob condições naturais, ocorre de forma aleatória, mas generalizada, em toda área. Entretanto, as atividades antrópicas podem adicionar materiais que contêm esses elementos aos solos, os quais podem atingir concentrações muito altas, que comprometem a qualidade dos ecossistemas (Davies \& Wixson, 1987; Chen et al., 1991; Holmgren et al., 1993).

Para atender aos requisitos impostos pela legislação vigente, os organismos de monitoramento ambiental necessitam de indicadores capazes de servir como referência para a avaliação continuada dos impactos ambientais causados pelas atividades antrópicas sobre os solos. Nesse sentido, as agências de proteção ambiental propõem o estabelecimento de valores orientadores que permitam identificar áreas poluídas ou contaminadas e, concomitantemente, avaliar o potencial de risco ao meio ambiente e à saúde humana (Cetesb, 2001; Soares, 2004).

Os valores de referência de qualidade (VRQ) ou de background são valores orientadores que representam a medida da concentração natural de elementos químicos em solos sem influência humana (Gough et al., 1994). Esses valores podem ser estabelecidos a partir da determinação dos teores naturais desses elementos no solo, levando-se em consideração a variação das classes e das propriedades físicas e químicas do solo. Dessa forma, vários estudos buscaram estabelecer os teores naturais de metais pesados em solos de regiões dos Estados Unidos, como na Flórida (Chen et al., 1999), Carolina do Sul (Gough et al., 1994), Ohio (Logan \& Miller, 1883), e de outros países, como Polônia (Dudka, 1992) e China (Chen et al., 1991).

No Brasil, com a crescente demanda social pela melhoria e manutenção da qualidade ambiental, 
realizaram-se alguns levantamentos dos teores naturais de metais pesados em solos com vistas a estabelecer valores orientadores para algumas regiões (Cetesb, 2001; Fadigas et al., 2002; Campos et al., 2003; Mineropar, 2005). Entretanto, ainda não foram definidos e regulamentados valores orientadores para os solos brasileiros.

Em vista do exposto, o presente estudo buscou obter os teores naturais de metais pesados e verificar a distribuição desses elementos nos solos de três bacias hidrográficas do Estado do Espírito Santo, objetivando o estabelecimento de Valores de Referência de Qualidade (VRQ).

\section{MATERIAL E MÉTODOS}

\section{Área de estudo}

A área de estudo foi estabelecida com base em aspectos geográficos, geoquímicos, pedológicos, importância socioeconômica e ambiental. Dessa forma, foram selecionadas as bacias hidrográficas Riacho, Reis Magos e Santa Maria da Vitória, no Estado do Espírito Santo (Figura 1).

A partir de uma análise conjunta dos mapas de solos e de geologia das três bacias hidrográficas, foram selecionados 56 locais para amostragem, de modo a se obter o maior número de ordens de solos (Quadro 1) e de agrupamentos litológicos representativos do Estado (Figura 2). Para isso, foram utilizados planos de informação, com feições do tipo polígono, obtidos a partir do mapa exploratório dos solos (Embrapa, 1978; IBGE, 2007), mapa geológico (Embrapa, 1978; IEMA, 2007) e o mapa de bacias hidrográficas do Estado do Espírito Santo (IEMA, 2007), todos na escala 1:750.000. Essas informações foram estruturadas em um Sistema de Informações Geográficas (SIG), e os

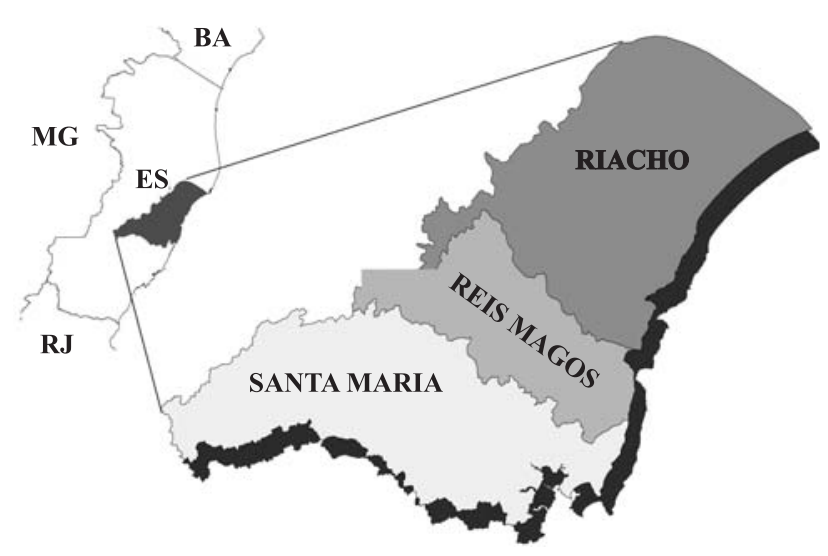

Figura 1. Localização das bacias hidrográficas Riacho, Reis Magos e Santa Maria da Vitória, no Estado do Espírito Santo.
Quadro 1. Ordens de solos representativas do Estado do Espírito Santo e respectivos locais de amostragem

\begin{tabular}{ll}
\hline Ordens de solo $^{(1)}$ & \multicolumn{1}{c}{ Locais de amostragem $^{(2)}$} \\
\hline Argissolo & $1,3,7,9,12,13,14,15,29,30,43,44,45$ e 56. \\
Cambissolo & $20,21,34,35,36$ e 52. \\
Gleissolo & $10,16,17,18,25,26$. \\
Espodossolo & 27,28 e 29. \\
Neossolo & $2,47,48,49$. \\
Latossolo & $4,5,6,8,11,19,22,23,24,32,33,37,38,39,40$, \\
& $42,43,47,50,51,53,54$ e 55.
\end{tabular}

(1) Classificação do primeiro nível taxonômico (EMBRAPA, 1999). ${ }^{(2)}$ Os locais de amostragem encontram-se plotados na figura 2 .

polígonos resultantes do processo de interseção representaram as unidades de mapeamento (UM).

\section{Coleta e preparo das amostras}

Para cada local de amostragem, foram colhidas amostras compostas de aproximadamente $2 \mathrm{~kg}$ de solo, coletadas com auxílio de trado holandês de aço inox, na profundidade de 0-0,2 m. Idealmente, elas foram coletadas em locais com mínima ou nenhuma ação antrópica, tal como proposto por Singh \& Steinnes (1994). As amostras coletadas foram secas ao ar, destorroadas, homogeneizadas e passadas em peneira de 2,0 mm de abertura de malha (ABNT 50), obtendose terra fina seca ao ar (TFSA).

\section{Caracterização química, física e métodos de extração dos teores totais de metais pesados}

As análises de caracterização física e química realizadas em todas as amostras coletadas foram: granulométrica, $\mathrm{pH}$ em $\mathrm{H}_{2} \mathrm{O}$ (Embrapa, 1997), capacidade de troca de cátions efetiva $\left(\mathrm{CTC}_{\mathrm{e}}\right)$ (Embrapa, 1999) e carbono orgânico total (CO) de acordo com Yeomans \& Bremner (1988), adaptado por Mendonça \& Matos (2005). Todas as análises foram realizadas em duplicata.

Os metais pesados determinados neste estudo foram selecionados com base no grau de toxidez (ATSDR, 2009) e naqueles mencionados com maior frequência como contaminantes em resíduos industriais e urbanos, insumos agrícolas ou subprodutos utilizados com finalidade corretiva e nutricional na agricultura (Malavolta, 1994).

Para extração dos teores totais de As, Cd, Co, Cr, $\mathrm{Cu}, \mathrm{Mn}, \mathrm{Mo}, \mathrm{Ni}, \mathrm{Pb}$ e $\mathrm{Zn}$, foram adotados procedimentos de ataque ácido, com digestão em forno microondas, marca CEM, modelo MDS 2000, conforme método SW-846 3052 da Environmental Protection Agency (USEPA, 2007). Frações de 0,250 g de solo foram, previamente, fragmentadas em almofariz de 


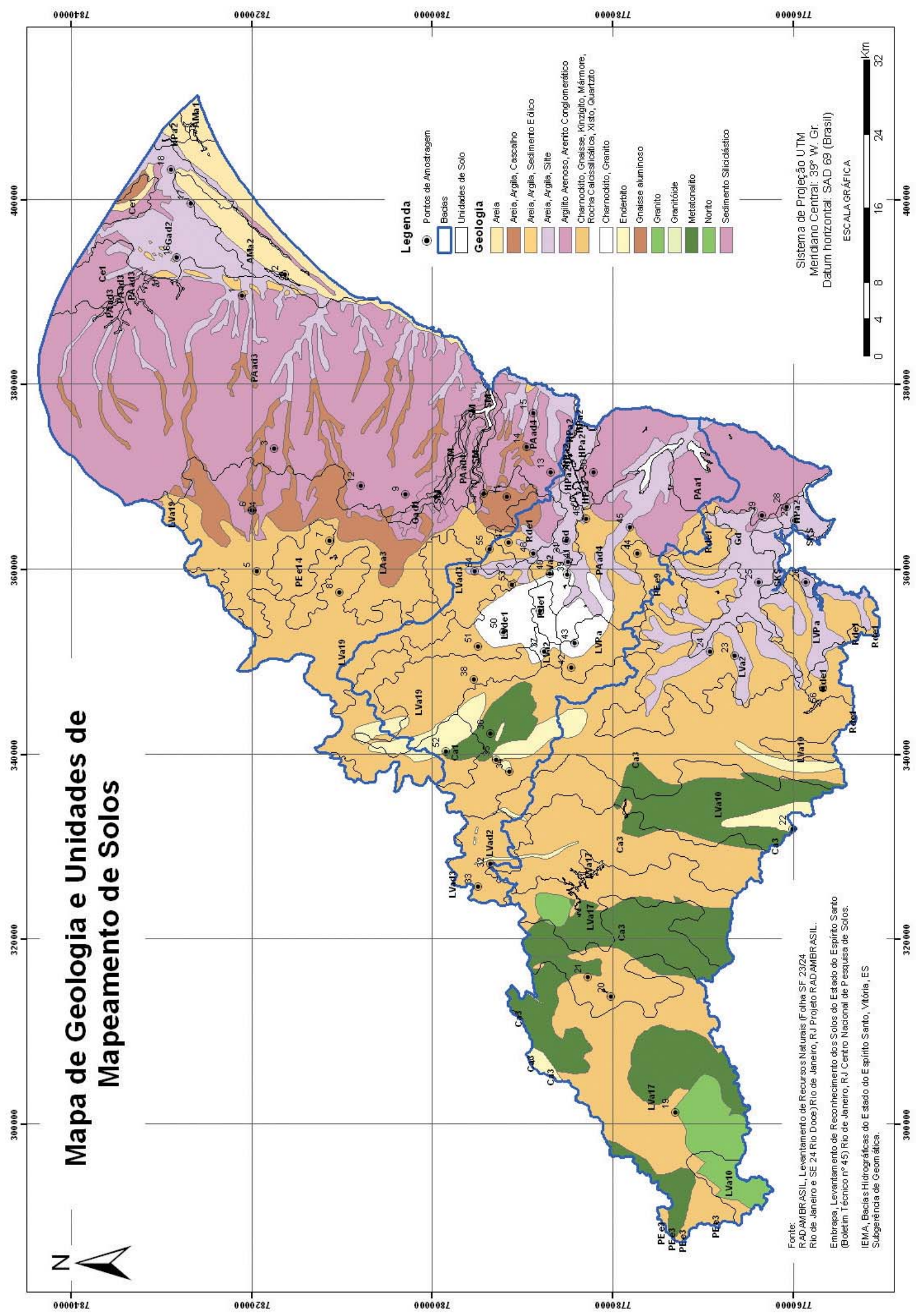

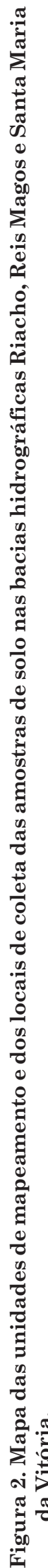


ágata, homogeneizadas e passadas em peneira de 0,149 mm de abertura de malha (ABNT 100). Posteriormente, foram colocadas em tubos de digestão de Teflon ${ }^{\circledR}$ de alta pressão, adicionando-se $9 \mathrm{~mL}$ de ácido nítrico $65 \%$ (v/v), 4 mL de ácido fluorídrico $36 \%$ (v/v) e $3 \mathrm{~mL}$ de ácido clorídrico $37 \%$ (v/v), todos de alta pureza analítica, aquecidos por radiação micro-ondas por 15 min a uma potência de $600 \mathrm{~W}$ a $180{ }^{\circ} \mathrm{C}$. Após resfriar, o extrato foi vertido para balões volumétricos de $50 \mathrm{~mL}$, completando-se o volume com solução de ácido bórico a $5 \%(\mathrm{~m} / \mathrm{v})$. Em seguida, o extrato foi transferido para tubos de $50 \mathrm{~mL}$ do tipo Falcon e centrifugados a $2.500 \mathrm{rpm}$ por $15 \mathrm{~min}$, sendo posteriormente armazenados em recipientes de polietileno em geladeira. As análises foram realizadas em duplicatas e, paralelamente, foram feitas provas em branco.

O controle de qualidade do método utilizado para análises dos teores totais de metais pesados foi avaliado por meio das taxas de recuperação de uma amostra de referência certificada SRM 2710-NIST (Montana Soil Highly ${ }^{\circledR}$ ).

\section{Determinação analítica}

A medida dos elementos químicos no extrato foi obtida por espectrofotometria de emissão óptica com plasma induzido (ICP-OES), marca Perkin Elmer, modelo Optima 3300 DV. As condições de operação do aparelho e alguns parâmetros associados às determinações realizadas por ICP-OES estão descritos nos quadro 2.

\section{Análise estatística}

Os resultados analíticos foram avaliados por análise descritiva, considerando os parâmetros de

Quadro 2. Comprimentos de onda selecionados e limites de deteç̧ão e quantificação obtidos

\begin{tabular}{cccc}
\hline Elemento & $\boldsymbol{\lambda}$ & $\mathbf{L D}^{(\mathbf{1})}$ & $\mathbf{L Q P}$ \\
& $\mathrm{nm}$ & $\mu \mathrm{g} \mathrm{L}^{-1}$ & $\mathrm{mg} \mathrm{kg}^{-1}$ \\
$\mathrm{Mn}$ & 259.37 & 0,32 & 0,06 \\
$\mathrm{Cr}$ & 267.71 & 2,34 & 0,47 \\
$\mathrm{Zn}$ & 213.85 & 3,24 & 0,65 \\
$\mathrm{~Pb}$ & 220.35 & 22,68 & 4,54 \\
$\mathrm{Ni}$ & 231.60 & 4,62 & 0,92 \\
$\mathrm{Cd}$ & 214.44 & 0,64 & 0,13 \\
$\mathrm{Cu}$ & 324.75 & 0,13 & 0,02 \\
$\mathrm{Mo}$ & 202.03 & 1,02 & 0,20 \\
$\mathrm{Co}$ & 228.61 & 2,02 & 0,40 \\
$\mathrm{Fe}$ & 238.20 & 6,07 & 1,21 \\
$\mathrm{Al}$ & 396.15 & 31,11 & 6,22 \\
$\mathrm{As}$ & 193.69 & 64,14 & 12,83 \\
$\mathrm{~V}$ & 290.88 & 4,62 & 0,92 \\
$\mathrm{Ti}$ & 334.94 & 1,22 & 0,24 \\
\hline
\end{tabular}

(1) Limite de detecção (LD): $3 \sigma\left(\operatorname{tg} \alpha^{-1}\right) .{ }^{(2)}$ Limite de quantificação praticável $(\mathrm{LQP})=\mathrm{LD}$ x fator diluição (FD). FD: 200 . posição, média e mediana e de dispersão, valores mínimos e máximos, desvio-padrão e coeficiente de variação. Os Valores de Referência de Qualidade (VRQ) foram estabelecidos com base nos percentis 75 e 90, conforme sugere o CONAMA (2010).

\section{RESULTADOS E DISCUSSÃO}

\section{Caracterização dos atributos físicos e químicos}

As amostras coletadas apresentavam características típicas de solos ácidos, com $\mathrm{pH}$ variando entre 4,85 e 5,21 (Quadro 3). Essas condições, de modo geral, favorecem a solubilização e mobilização de metais pesados na solução do solo e, por extensão, as perdas desses elementos por lixiviação. Por outro lado, a argila e a matéria orgânica influenciam na retenção da grande maioria dos metais pesados no solo (Alleoni et al., 2005; Guilherme et al., 2005). As amostras apresentaram em média 3,64 dag $\mathrm{kg}^{-1}$ de $\mathrm{CO}$ total, 52,20 dag kg-1 de areia e 49,40 dag kg-1 de silte + argila, o que caracteriza solos de textura argiloarenosa. A $\mathrm{CTC}_{\mathrm{e}}$ mostrou-se diferenciada apenas para os Gleissolos, os quais apresentaram os maiores valores médios em relação às demais ordens. Esse comportamento diferenciado deve-se, principalmente, aos altos teores de $\mathrm{CO}$ e de argila para a maioria das amostras dessa ordem de solo.

\section{Teores naturais de metais pesados nos solos}

Os teores totais extraídos para os elementos As, $\mathrm{Cd}$, Co, Cr, Cu, Mn, Mo, Ni, Pb e Zn nos solos das bacias hidrográficas Riacho, Reis Magos e Santa Maria da Vitória são relativamente baixos e inferiores aos reportados para solos em outros países, com exceção do As, Cr e Mo, que apresentaram teores ligeiramente superiores aos valores reportados para os Estados Unidos (Quadro 4). Campos et al. (2003), trabalhando com 19 Latossolos, encontraram teores de $\mathrm{Cd}$ $\left(0,66 \mathrm{mg} \mathrm{kg}^{-1}\right), \mathrm{Cu}\left(65 \mathrm{mg} \mathrm{kg}^{-1}\right), \mathrm{Ni}\left(18 \mathrm{mg} \mathrm{kg}^{-1}\right), \mathrm{Pb}$ $\left(22 \mathrm{mg} \mathrm{kg}^{-1}\right)$ e $\mathrm{Zn}\left(39 \mathrm{mg} \mathrm{kg}^{-1}\right)$ superiores aos determinados para os solos do Espírito Santo. Um levantamento geoquímico multielementar foi realizado no Estado do Paraná (Mineropar, 2005), e os teores de metais pesados obtidos nesse estudo também foram superiores aos encontrados nos solos do Espírito Santo (Quadro 4). No entanto, algumas semelhanças nos teores obtidos para As (7,25 $\left.\mathrm{mg} \mathrm{kg}^{-1}\right), \mathrm{Cd}\left(0,18 \mathrm{mg} \mathrm{kg}^{-1}\right)$ e Mo (1,68 $\left.\mathrm{mg} \mathrm{kg}^{-1}\right)$ são observadas.

As diferenças entre os teores naturais de metais pesados em solos são atribuídas, principalmente, ao material de origem e a fatores pedogenéticos (Moura, 1985; Davies \& Wixson, 1987; Oliveira et al., 1998; Guilherme et al., 2005, Alleoni et al., 2005). Essa afirmação concorda, até certo ponto, com os baixos teores encontrados nos solos das três bacias hidrográficas, visto que aproximadamente dois terços 
Quadro 3. Caracterização física e química das amostras de solo

\begin{tabular}{|c|c|c|c|c|c|}
\hline Ordem de solo $^{(1)}$ & $\mathrm{pH} \mathrm{H}_{2} \mathrm{O}$ & Areia & Silte + Argila & $\mathrm{CO}$ & CTCe \\
\hline & & & - dag $\mathrm{kg}^{-1(3)}$ & & $\mathrm{cmol}_{\mathrm{c}} \mathrm{dm}^{-3}$ \\
\hline Argissolos $(\mathrm{n}=14)^{(2)}$ & $4,91 \pm 0,48$ & $54,85 \pm 9,69$ & $45,15 \pm 9,69$ & $1,98 \pm 0,65$ & $2,96 \pm 1,29$ \\
\hline Cambissolos $(\mathrm{n}=6)$ & $4,92 \pm 0,36$ & $62,50 \pm 7,77$ & $37,50 \pm 7,77$ & $3,34 \pm 1,64$ & $2,57 \pm 0,51$ \\
\hline Espodossolos $(\mathrm{n}=3)$ & $4,85 \pm 0,22$ & $56,67 \pm 40,72$ & $43,33 \pm 40,72$ & $3,68 \pm 2,00$ & $8,80 \pm 10,00$ \\
\hline Gleissolos $(n=6)$ & $5,21 \pm 1,11$ & $31,42 \pm 26,70$ & $68,58 \pm 26,70$ & $9,73 \pm 7,42$ & $8,37 \pm 5,81$ \\
\hline Latossolos $(\mathrm{n}=23)$ & $4,92 \pm 0,51$ & $52,96 \pm 11,76$ & $47,04 \pm 11,76$ & $1,90 \pm 0,80$ & $2,89 \pm 1,04$ \\
\hline Neossolos $(\mathrm{n}=4)$ & $5,02 \pm 0,28$ & $71,50 \pm 17,93$ & $28,50 \pm 15,53$ & $1,22 \pm 0,40$ & $3,50 \pm 2,11$ \\
\hline \multicolumn{6}{|l|}{ Todos os solos (4) } \\
\hline Média & 4,97 & 52,20 & 49,40 & 3,64 & 4,84 \\
\hline Mediana & 4,92 & 54,85 & 46,08 & 2,66 & 3,23 \\
\hline Maior & 5,21 & 62,50 & 68,57 & 9,73 & 8,8 \\
\hline Menor & 4,85 & 31,42 & 37,5 & 1,22 & 2,57 \\
\hline Desvio-padrão & 0,12 & 10,70 & 10,94 & 3,12 & 2,91 \\
\hline $\mathrm{CV}(\%)$ & 2,59 & 20,49 & 22,16 & 85,80 & 60,08 \\
\hline
\end{tabular}

(1) Classificação do primeiro nível taxonômico (Embrapa, 1999). ${ }^{(2)}$ Número de amostras de cada classe de solo. ${ }^{(3)}$ Média aritmética \pm desvio-padrão. ${ }^{(4)}$ Atributos relativos ao conjunto de 56 amostras de solo.

Quadro 4. Teores totais de metais pesados para solos do Espírito Santo em comparação com dados compilados da literatura

\begin{tabular}{|c|c|c|c|c|c|c|c|c|}
\hline \multirow{2}{*}{ Elemento } & \multicolumn{3}{|c|}{ Valor para o Espírito Santo } & \multicolumn{5}{|c|}{ Valor Internacional $^{(1)}$} \\
\hline & $\mathrm{NS}^{(2)}$ & Média & CV & $\mathbf{P R}^{(3)}$ & China & USA & Austrália & $\mathrm{WS}^{(4)}$ \\
\hline & & $\mathrm{mg} \mathrm{kg}^{-1}$ & $\%$ & & & - $\mathrm{mg} \mathrm{kg}$ & & \\
\hline As & 44 & 6,80 & 94,55 & 7,25 & 9,20 & 5,20 & 20,00 & $\mathrm{NA}^{(5)}$ \\
\hline $\mathrm{Cd}$ & 0 & $<\mathrm{LD}$ & - & 0,18 & 0,07 & 1,60 & 1,00 & 0,40 \\
\hline Co & 51 & 8,64 & 54,81 & 23,84 & NA & 20,00 & NA & 12,80 \\
\hline $\mathrm{Cr}$ & 55 & 41,07 & 51,69 & 105,00 & 53,90 & 37,00 & 100,00 & 50,00 \\
\hline $\mathrm{Cu}$ & 50 & 5,57 & 123,29 & 141,72 & 20,00 & 17,00 & 100 & 12,00 \\
\hline $\mathrm{Mn}$ & 56 & 131,69 & 89,60 & 736,84 & 482,00 & 330,00 & NA & 450,00 \\
\hline Mo & 56 & 1,43 & 137,57 & 1,68 & 1,20 & 0,59 & NA & 1,50 \\
\hline $\mathrm{Ni}$ & 54 & 6,65 & 96,47 & 34,16 & 23,40 & 13,00 & 60,00 & 25,00 \\
\hline $\mathrm{Pb}$ & 27 & 8,79 & 158,62 & 23,21 & 23,60 & 16,00 & 150,00 & 15,00 \\
\hline $\mathrm{Zn}$ & 52 & 22,61 & 87,27 & 75,26 & 67,70 & 48,00 & $1.400,00$ & 40,00 \\
\hline
\end{tabular}

${ }^{(1)}$ Citados por Chen et al. (1991) e Guilherme et al. (2005). ${ }^{(2)}$ Corresponde ao número de amostras de solo que apresentaram valores acima de zero, em um total de 56 amostras. Apesar da incerteza, no cálculo da média foram considerados valores positivos abaixo do LQP do método para As (35 amostras < LQP > 0), Pb (13 amostras < LQP > 0), Mo (7 amostras < LQP > 0) e Ni (4 amostras $<$ LQP $>0$ ). ${ }^{(3)}$ Citados por MINEROPAR (2005) para solos do Estado do Paraná. ${ }^{(4)}$ Solos do mundo. ${ }^{(5)}$ Dados não avaliados.

da área do Estado são ocupados por rochas Cristalinas Pré-Cambrianas, e o restante, por um manto de sedimentos Terciários e Quaternários (Embrapa, 1978). Solos derivados de rochas sedimentares e ígneas ácidas cristalinas, via de regra, apresentam teores bem menores de metais pesados, quando comparados aos solos originados de rochas básicas, especialmente as máficas, que são naturalmente mais ricas em metais.

Por outro lado, a influência da pedogênese não é óbvia, considerando-se que os teores médios de metais pesados variaram amplamente entre as amostras de classes diferentes e também entre solos pertencentes a uma mesma classe, conforme se verifica pelos valores do coeficiente de variação para os teores dos elementos avaliados (Quadro 5). Essas variações nos teores médios de metais pesados em solos também foram observadas por outros autores, como Amaral Sobrinho et al. (1997) e Fadigas et al. (2006), os quais justificam essas diferenças em função das variações nas suas propriedades químicas e físicas.

Os teores de Cd ficaram abaixo do limite de detecção (LD) do método para todas as amostras de solos (Quadros 5 e 6). O mesmo foi observado pela Cetesb (2001) para solos do Estado de São Paulo. Esse fato está relacionado à abundância desse elemento na natureza. $\mathrm{O}$ teor total médio de $\mathrm{Cd}$ na crosta terrestre 
Quadro 5. Teores totais médios e coeficiente de variação para metais pesados em seis ordens de solo no Estado do Espírito Santo ${ }^{(1)}$

\begin{tabular}{|c|c|c|c|c|c|c|}
\hline \multirow{2}{*}{ Elemento } & \multicolumn{2}{|c|}{ Argissolo } & \multicolumn{2}{|c|}{ Cambissolo } & \multicolumn{2}{|c|}{ Gleissolo } \\
\hline & Média & CV & Média & $\mathrm{CV}$ & Média & $\mathrm{CV}$ \\
\hline & $\mathrm{mg} \mathrm{kg}^{-1}$ & $\%$ & $\mathrm{mg} \mathrm{kg}^{-1}$ & $\%$ & $\mathrm{mg} \mathrm{kg}^{-1}$ & $\%$ \\
\hline As & 7,32 & 78,70 & 3,13 & 139,04 & 6,77 & 70,53 \\
\hline $\mathrm{Cd}$ & $<\mathrm{LD}^{(2)}$ & - & $<\mathrm{LD}$ & - & $<\mathrm{LD}$ & \\
\hline Co & 9,80 & 29,36 & 4,45 & 35,88 & 6,50 & 45,81 \\
\hline $\mathrm{Cr}$ & 39,65 & 43,22 & 51,33 & 52,97 & 51,89 & 46,95 \\
\hline $\mathrm{Cu}$ & 2,77 & 105,76 & 5,13 & 71,64 & 16,61 & 97,68 \\
\hline $\mathrm{Mn}$ & 100,31 & 51,14 & 119,56 & 57,67 & 87,51 & 84,48 \\
\hline Mo & 2,48 & 109,00 & 0,50 & 80,16 & 2,47 & 72,26 \\
\hline $\mathrm{Ni}$ & 4,35 & 84,67 & 7,97 & 119,12 & 11,19 & 88,91 \\
\hline $\mathrm{Pb}$ & 6,22 & 82,30 & $<\mathrm{LD}$ & - & 7,08 & 65,12 \\
\hline $\mathrm{V}$ & 90,10 & 21,06 & 102,51 & 13,51 & 77,97 & 33,24 \\
\hline \multirow[t]{2}{*}{$\mathrm{Zn}$} & 17,91 & 90,51 & 13,60 & 70,81 & 15,56 & 60,12 \\
\hline & \multicolumn{2}{|c|}{ Espodossolo ${ }^{(3)}$} & \multicolumn{2}{|c|}{ Neossolo } & \multicolumn{2}{|c|}{ Latossolo } \\
\hline As & 1,38 & 100,47 & 17,51 & 50,19 & 5,68 & 91,58 \\
\hline $\mathrm{Cd}$ & $<\mathrm{LD}$ & - & $<\mathrm{LD}$ & & $<\mathrm{LD}$ & - \\
\hline Co & 17,56 & 0 & 10,70 & 68,30 & 9,06 & 61,98 \\
\hline $\mathrm{Cr}$ & 34,68 & 59,41 & 15,68 & 84,87 & 40,58 & 50,49 \\
\hline $\mathrm{Cu}$ & 5,18 & 111,67 & 2,99 & 72,13 & 5,08 & 75,56 \\
\hline $\mathrm{Mn}$ & 69,92 & 107,36 & 276,76 & 89,98 & 148,30 & 85,63 \\
\hline Mo & 3,46 & 120,12 & 0,40 & 41,72 & 0,69 & 106,01 \\
\hline $\mathrm{Ni}$ & 8,75 & 124,54 & 4,24 & 57,13 & 6,46 & 79,81 \\
\hline $\mathrm{Pb}$ & 1,14 & 0 & 14,64 & 104,79 & 10,81 & 196,04 \\
\hline V & 57,60 & 102,74 & 55,29 & 71,63 & 102,25 & 33,96 \\
\hline $\mathrm{Zn}$ & 47,87 & 117,21 & 37,32 & 80,61 & 25,84 & 74,22 \\
\hline
\end{tabular}

(1) Classificação do primeiro nível taxonômico (Embrapa, 1999). ${ }^{(2)}$ Teores abaixo do limite de detecção (LD) do método. ${ }^{(3)}$ Para o elemento $\mathrm{Co} \mathrm{e} \mathrm{Pb}$, o valor apresentado foi obtido a partir de uma amostra. As demais amostras apresentaram teores < LD para esses elementos.

é de aproximadamente 0,15 $\mathrm{mg} \mathrm{kg}^{-1}$ (Bowen, 1979; Heinrichs et al., 1980), e nos solos a média é de $0,06 \mathrm{mg} \mathrm{kg}^{-1}$ (EPA, 1992). Ross (1994) verificou que o teor desse elemento é maior para os solos que tenham sua origem associada às rochas máficas e restrita para os solos originados de gnaisse e arenito + sedimentos do Terciário. Além disso, o $\mathrm{pH}$ altera diretamente a dinâmica do Cd no solo (Naidu et al., 1994). Sob condições ácidas, o Cd aumenta sua solubilidade e é muito pouco adsorvido pelos coloides orgânicos e minerais do solo, principalmente devido à sua baixa afinidade com óxidos de Fe e Mn (Ross, 1994; KabataPendias \& Pendias, 2001). Nessas condições, a mobilidade desse elemento e as perdas por lixiviação podem ser determinantes para os teores no solo, conforme ressaltado por autores como Pardo (2000) e Oliveira \& Matiazzo (2001). Todos esses fatores contribuem para os baixos teores de Cd nos solos do Espírito Santo em condições naturais. Os demais metais pesados tiveram resultados analíticos abaixo do LD inferiores a $10 \%$.

$\mathrm{O} \mathrm{Pb}$ apresentou $75 \%$ e o As, $84 \%$, dos resultados analíticos abaixo do limite de quantificação praticável (LQP) do método. Apesar disso, os teores médios desses elementos nas amostras de solo com valores acima de zero, incluindo-se aí várias amostras com valores $<\mathrm{LQP}$, ficaram bem próximos dos teores médios reportados pela Environmental Protection Agency (USEPA, 1992) para $\mathrm{Pb}\left(10 \mathrm{mg} \mathrm{kg}^{-1}\right)$ e para As (5 $\mathrm{mg} \mathrm{kg}^{-1}$ ).

Os valores de recuperação de metais pesados para solubilização total das amostras do material de referência certificado variaram de 81 a $108 \%$

Quadro 6. Teores totais recuperados para o material de referência certificado (SRM 2710 - NIST) pelo método SW 846 EPA 3052

\begin{tabular}{ccc}
\hline Elemento & Teor total $^{(1)}$ & Recuperação( $^{(2)}$ \\
\hline & mg kg-1(2) & $\%$ \\
$\mathrm{As}$ & $612,31 \pm 29,76$ & 97,81 \\
$\mathrm{Cd}$ & $23,57 \pm 0,03$ & 108,13 \\
$\mathrm{Co}$ & $10,05 \pm 0,39$ & 100,54 \\
$\mathrm{Cr}$ & $36,23 \pm 0,21$ & 92,88 \\
$\mathrm{Cu}$ & $3034,91 \pm 38,93$ & 102,87 \\
$\mathrm{Mn}$ & $8846,09 \pm 190,91$ & 87,59 \\
$\mathrm{Mo}$ & $19,55 \pm 0,20$ & 102,90 \\
$\mathrm{Ni}$ & $11,68 \pm 1,03$ & 81,67 \\
$\mathrm{~Pb}$ & $5243,75 \pm 101,66$ & 94,79 \\
$\mathrm{~V}$ & $75,17 \pm 1,97$ & 98,13 \\
$\mathrm{Zn}$ & $6755,04 \pm 45,79$ & 97,17 \\
\hline
\end{tabular}

(1) Média aritmética \pm desvio-padrão. ${ }^{(2)} \%$ Recuperação = (valor recuperado/valor certificado) x 100 . 
(Quadro 6), encontrando-se dentro da faixa recomendada para a maioria dos elementos estudados (USEPA, 1996). Portanto, os resultados de recuperação dos analitos na amostra certificada atestam a qualidade das análises.

\section{Estabelecimento de valores de referência de qualidade (VRQ)}

A tendência mundial sugere a adoção dos VRQ para metais pesados em solos como uma primeira etapa nas ações de monitoramento da qualidade ambiental. Daí a importância de esses valores serem estabelecidos, sempre que possível, com base em dados locais ou em áreas com características pedogeoquímicas semelhantes àquelas em estudo (Cetesb, 2001; Borges Jr. et al., 2002). No quadro 7 são apresentados os VRQ para solos do Estado do Espírito Santo, obtidos a partir dos percentis 75 (quartil superior) e 90 dos teores totais para 10 metais pesados.

De modo geral, os VRQ obtidos pelo quartil superior (Quadro 7) foram semelhantes aos valores reportados por Su \& Yang (2008) para solos da Ásia em relação a $\mathrm{Cr}\left(55,8 \mathrm{mg} \mathrm{kg}^{-1}\right), \mathrm{Zn}\left(33,7 \mathrm{mg} \mathrm{kg}^{-1}\right) \mathrm{e}$ $\mathrm{Cu}\left(5,1 \mathrm{mg} \mathrm{kg}^{-1}\right)$. Os valores obtidos por esses autores para $\mathrm{Mn}\left(404.8 \mathrm{mg} \mathrm{kg}^{-1}\right)$, Ni $\left(17,7 \mathrm{mg} \mathrm{kg}^{-1}\right)$ e $\mathrm{Pb}$ (15,5 mg kg-1) foram superiores, e para As (5,2 $\left.\mathrm{mg} \mathrm{kg}^{-1}\right)$ e Co (5,7 $\left.\mathrm{mg} \mathrm{kg}^{-1}\right)$, inferiores aos resultados obtidos para os solos do Estado do Espírito Santo. Estudos conduzidos por Ma et al. (1997) e Chen et al. (1999) para solos da Flórida mostram que os VRQ obtidos para os solos do Estado do Espírito Santo encontramse dentro do intervalo de confiança estabelecido por esses autores para a maioria dos metais pesados analisados. Em relação aos VRQ propostos por Fadigas et al. (2006) para solos brasileiros, observou-se que os valores do quartil superior médio entre os grupos formados para $\mathrm{Cr}\left(41 \mathrm{mg} \mathrm{kg}^{-1}\right)$ e Co $\left(8 \mathrm{mg} \mathrm{kg}^{-1}\right)$ estão ligeiramente abaixo dos estabelecidos para o Estado do Espírito Santo (Quadro 7). Os valores obtidos por Fadigas et al. (2006) para Ni $\left(17 \mathrm{mg} \mathrm{kg}^{-1}\right), \mathrm{Cu}$ $\left(25 \mathrm{mg} \mathrm{kg}^{-1}\right)$, Cd $\left(0,8 \mathrm{mg} \mathrm{kg}^{-1}\right)$ e Pb $\left(20 \mathrm{mg} \mathrm{kg}^{-1}\right)$ apresentam-se acima e, para o $\mathrm{Zn}\left(30 \mathrm{mg} \mathrm{kg}^{-1}\right)$, aproxima-se muito do valor obtido para o Estado do Espírito Santo. Essas diferenças nos teores médios e nos valores de referência de qualidade para metais pesados são comuns nesse tipo de estudo, em razão das diferenças de composição mineralógica do material de origem dos solos e das influências climáticas nos processos pedogenéticos (Tack et al., 1997; De Temmerman et al., 2003). Características dos solos como teor e composição da fração argila e conteúdo de matéria orgânica, as condições físico-químicas e as diferenças entre técnicas analíticas empregadas nas determinações também contribuem para a variabilidade dos resultados (Amaral Sobrinho et al.,1997; Fadigas et al., 2006).

A despeito das diferenças metodológicas e das condições geoclimáticas e geomorfológicas, os VRQ obtidos neste estudo estão bem próximos ou abaixo daqueles publicados pela Cetesb (2001), dos obtidos por Fadigas et al. (2006) e também em relação a valores internacionais. Vale salientar que os valores obtidos para o Estado de São Paulo (Cetesb, 2001) não representam teoricamente os teores totais, posto que foram extraídos com água-régia. Também os resultados de Fadigas et al. (2006) reúnem dados obtidos a partir de extração com água-régia e digestão nitroperclórica, considerados teores "pseudototais". Ainda assim, os teores totais para os solos do Estado do Espírito Santo não produziram valores de referência substancialmente superiores aos obtidos por esses autores. Esses resultados sugerem valores comparáveis entre teores totais e pseudototais. Provavelmente, as diferenças entre os teores totais, obtidos por digestão fluorídrica, e os teores pseudototais, obtidos por água-régia, não são relevantes em solos bastante intemperizados, como os Latossolos e Argissolos, que são as ordens de solo dominantes no Brasil e também no Estado do Espírito Santo. Isso porque, nessas condições, a fração areia dos solos é dominada por quartzo e já não restam muitos metais dentro das estruturas dos silicatos (Lima et al., 2005). A tendência é de acúmulo de metais na fração argila, associados às fases mais oxídicas ou orgânicas dos solos. Por outro lado, em solos mais jovens, de textura mais grosseira, podem-se esperar maiores diferenças entre teores totais e pseudototais, a depender da mineralogia das frações areia e silte.

\section{VRQ a partir dos percentis 75 e 90}

A resolução do CONAMA n ${ }^{\circ}$ 420/2009 (CONAMA, 2010) estabelece que o VRQ possa ser obtido com base no percentil 75 ou 90 do universo amostral. Assim, ao considerar o percentil 90 (P 90) para o estabelecimento do VRQ para o Estado do Espírito Santo, o valor praticamente dobra em relação ao

Quadro 7. Valores de referência de qualidade para solos no Estado do Espírito Santo

\begin{tabular}{|c|c|c|c|c|c|c|c|c|c|c|}
\hline & As & $\mathbf{C d}$ & Co & $\mathrm{Cr}$ & $\mathbf{C u}$ & Mn & Mo & $\mathrm{Ni}$ & $\mathbf{P b}$ & $\mathrm{Zn}$ \\
\hline & & & & \multicolumn{2}{|c|}{$\ldots \mathrm{mg} \mathrm{kg}^{-1} \_$} & & & & & \\
\hline Média & 8,14 & $<\mathrm{LQ}$ & 7,90 & 40,34 & 4,98 & 131,69 & 1,44 & 6,44 & 5,39 & 21,02 \\
\hline Máximo & 28,31 & $<\mathrm{LQ}$ & 25,39 & 103,97 & 40,05 & 602,38 & 10,23 & 25,60 & 70,69 & 87,54 \\
\hline Mínimo & 6,41 & $<\mathrm{LQ}$ & 0,20 & 0,23 & 0,01 & 5,56 & 0,10 & 0,46 & 2,27 & 0,32 \\
\hline P 75 & $<12,83$ & $<0,13$ & 10,21 & 54,13 & 5,91 & 137,80 & 1,74 & 9,17 & $<4,54$ & 29,87 \\
\hline P 90 & 14,28 & $<0,13$ & 14,56 & 68,81 & 10,78 & 253,01 & 3,36 & 17,22 & 8,92 & 49,32 \\
\hline
\end{tabular}


quartil superior (P 75), para a maioria dos metais pesados analisados (Quadro 7), com exceção para Cr e Co. Isso significa que, ao ser mais restritivo tendo em vista maior segurança ambiental, o número de casos de solos suspeitos de contaminação aumenta, o que obviamente traz desvantagens para o gerenciamento das áreas contaminadas por parte dos órgãos ambientais. No entanto, isso nem sempre se traduz em maior qualidade ambiental, posto que os dados para obtenção dos VRQ são obtidos a partir de áreas preservadas ou minimamente impactadas por efeito antrópico. Em consequência, a grande maioria dos valores que extrapolam o quartil superior (P 75), provavelmente, não representa deterioração da qualidade ambiental dos solos. Por essa razão, é mais razoável adotar o valor menos restritivo (P 90) ou adotar vários valores dentro de um mesmo Estado, a partir de grupos de solos compatíveis quanto às suas propriedades, agrupados por critérios, de preferência pedogeoclimáticos e pedogeomorfológicos, com base em métodos estatísticos adequados.

\section{CONCLUSÕES}

1. Os teores totais extraídos para os elementos As, $\mathrm{Cd}, \mathrm{Co}, \mathrm{Cr}, \mathrm{Cu}, \mathrm{Mn}, \mathrm{Mo}, \mathrm{Ni}, \mathrm{Pb}$ e $\mathrm{Zn}$ nos solos das bacias hidrográficas do Riacho, Reis Magos e Santa Maria da Vitória são relativamente baixos quando comparados aos obtidos em solos de outros Estados brasileiros e em outros países.

2. Os teores totais de metais pesados obtidos para os solos do Espírito Santo não produziram valores de referência substancialmente superiores aos obtidos para solos brasileiros e para solos em outros países.

3. A adoção dos VRQ a partir do percentil 90 traz vantagens para o gerenciamento de áreas contaminadas, por parte dos órgãos ambientais, sem comprometer a qualidade ambiental dos solos.

\section{AGRADECIMENTOS}

À Coordenação de Aperfeiçoamento Pessoal de Nível Superior (CAPES), pela concessão da bolsa de estudo, e ao Instituto de Pesquisa, Assistência Técnica e Extensão Rural (Incaper), pelo apoio logístico na coleta das amostras de solo.

\section{LITERATURA CITADA}

AGENCY FOR TOXIC SUBSTANCES E DISEASE REGISTRY - ATSDR. 2007 CERCLA Priority List of Hazardous Substances. Disponível em: < http://www.atsdr.cdc.gov/ cercla/07list.htm >. Acesso em 15 de set. 2009.
ALLEONI, L.R.F.; BORBA, R.P. \& CAMARGO, O.A. Metais pesados: Da cosmogênese aos solos brasileiros. In: TORRADO-VIDAL, P.; ALLEONI, L.R.F.; COOPER, M. \& SILVA, A.P., eds. Tópicos em ciência do solo. Viçosa, MG, Sociedade Brasileira de Ciência do Solo, 2005. v.4. p.1- 42 .

AMARAL SOBRINHO, N.M.B.; VELLOSO, A.C.X. \& de OLIVEIRA, C. Solubilidade de metais pesados em solo tratado com resíduo siderúrgico. R. Bras. Ci. Solo, 21:916, 1997.

BORGES JR., M. Distribuição e contaminação de $\mathrm{Zn} \mathrm{Cd} \mathrm{e} \mathrm{Pb}$ em perfis de solo e obtenção de valores de referência local no município de Vazante - MG. Viçosa, MG, Universidade Federal de Viçosa, 2002. 152p. (Tese de Doutorado)

BOWEN, H.J.M. Environmental chemistry of the elements. London, Academic Press, 1979. 176p.

CAMPOS, M.L.; PIERANGELI, M.A.P.; GUILHERME, L.R.G.; MARQUES, J.J.G.S.M. \& CURI, N. Baseline concentration of heavy metals in Brazilian Latosols. Comm. Soil Sci. Plant. Anal., 34:547-557, 2003.

CHEN, J.; WEI, F.; ZHENG, C.; WU, Y. \& ADRIAN, D.C. Background concentrations of elements in soils of China. Water Air Soil Poll., 57-58:699-712, 1991.

CHEN, M.; MA, L.Q. \& HARRIS, W.G. Baseline concentrations of 15 trace elements in Florida surface soils. J. Environ. Qual., 28:1173-1181, 1999.

COMPANHIA DE TECNOLOGIA DE SANEAMENTO AMBIENTAL - CETESB. Relatório de estabelecimento de valores orientadores para solos e águas subterrâneas no Estado de São Paulo. São Paulo, Cetesb, 2001. 247p. (Relatório Técnico)

CONSELHO NACIONAL DO MEIO AMBIENTE - CONAMA. Resolução $\mathrm{n}^{\circ}$ 420/2009. Disponível em < http:// www.mma.gov.br/port/conama/legiabre.cfm.htm > Acesso em 20 de maio 2010.

DAVIES, B.E. \& WIXSON, B.G. Use of factor analysis to differentiate pollutants from other trace metals in surface soils of the mineralized area of Madison County, Missouri, USA. Water Air Soil Poll., 33:339-348, 1987.

DE TEMMERMAN, L.; VANONGEVAL, L.; BOON, W. \& HOENIG, G. Heavy metal content of arable soils in northern Belgium. Water Air Soil Poll., 148:61-73, 2003.

DUDKA, S. Factor analysis of total element concentrations in surface soils of Poland. Sci Total Environ., 121:39-52, 1992.

EMPRESA BRASILEIRA DE PESQUISA AGROPECUÁRIA EMBRAPA. Serviço nacional de levantamento e conservação de solo. Rio de Janeiro, Centro Nacional de Pesquisa de Solos, 1978. 461p. (Boletim Técnico, 45)

EMPRESA BRASILEIRA DE PESQUISA AGROPECUÁRIA EMBRAPA. Manual de métodos de análises de solos. 2.ed. Rio de Janeiro, Centro Nacional de Pesquisa de Solos, 1997. 212p. 
EMPRESA BRASILEIRA DE PESQUISA AGROPECUÁRIA EMBRAPA. Manual de métodos de análises químicas de solos, plantas e fertilizantes. Rio de Janeiro, Centro Nacional de Pesquisa de Solos, 1999. 370p.

EMPRESA BRASILEIRA DE PESQUISA AGROPECUÁRIA EMBRAPA. Sistema brasileiro de classificação de solos. Rio de Janeiro, Centro Nacional de Pesquisa de Solos, 1999. 412p.

FADIGAS, F.S.; SOBRINHO, N.M.B.A.; MAZUR, N.; ANJOS, L.H.C. \& FREIXO, A.A. Concentrações naturais de metais pesados em algumas classes de solos brasileiros. Bragantia, 61:151-159, 2002.

FADIGAS, F.S.; SOBRINHO, N.M.B.A.; MAZUR, N.; ANJOS, L.H.C. \& FREIXO, A.A. Proposição de valores de referência para a concentração natural de metais pesados em solos. R. Bras. Eng. Agric. Amb., 10:699-705, 2006.

GOUGH, L.P.; SEVERSON, R.C. \& JACKSON, L.L. Baseline element concentrations in soils and plants, Bull Island, Cape Romain National Wildlife Refuge, South Carolina, USA. Water Air Soil Poll., 74:1-17, 1994.

GUILHERME, L.R.G.; MARQUES, J.J.; PIERANGELI, M.A.P.; ZULIANI, D.Q.; CAMPOS, M. L. \& MARCHI, G. Elementos-traço em solos e sistemas aquáticos. In: TORRADO-VIDAL, P.; ALLEONI, L.R.F.; COOPER, M. \& SILVA, A.P., eds. Tópicos em ciência do solo. Viçosa, MG, Sociedade Brasileira de Ciência do Solo, 2005. v.4. p.345-390.

HEINRICHS, H.; SCHULTZ-DOBRICK, B. \& WEDEPOHL, K.H. Terrestrial geochemistry of CD, BI, TL, PB, ZN and RB. Geochim. Cosmochim. Acta, 44:1519-1532, 1980.

HOLMGREN, G.S.; MEYER, M.W.; CHANEY, R.L. \& DANIELS, R.B. Cadmium, lead, zinc, copper, and nickel in agricultural soils of the United States of America. J. Environ. Qual., 22:335-348, 1993.

INSTITUTO BRASILEIRO DE GEOGRAFIA E ESTATÍSTICA - IBGE/EMPRESA BRASILEIRA DE PESQUISA AGROPECUARIA - EMBRAPA. Serviço Nacional de Levantamento e Conservação de Solos (Rio de Janeiro, RJ). Mapa exploratório dos solos do estado Espírito Santo. RadamBrasil, 1985. Disponível em: <http:// biblioteca.ibge.gov.br. htm>. Acesso em 20 ago. 2007.

INSTITUTO ESTADUAL DO MEIO AMBIENTE E RESURSOS HIDRICOS DO ESPÍRITO SANTO - IEAMA. Subgerência de geomática. Disponível em: <http:// www.iema.es.gov.br/default.asp.htm>. Acesso em 20 ago. 2007.

KABATA-PENDIAS, A. \& PENDIAS, H. Trace elements in soils and plants. 3.ed. Boca Raton, CRC Press, 2001. 413p.

LIMA, E.A.R.; LIMA, W.N.; SIQUEIRA, G.W. \& SIQUEIRA, N.V.M. Influência da mineralogia na composição química de metais pesados nos sedimentos pelíticos superficiais da Plataforma Continental do Amazonas. Geoquim. Bras., 19:103-117, 2005.

LOGAN, T.J. \& MILLER R.H. Background levels of heavy metals in Ohio farm soils. Soil contamination analysis. Res. Circ. Ohio Agric. Res. Dev. Cent., 275:3-15, 1983.
MA, L.Q.; TAN, F. \& HARRIS, W.G. Concentrations and distributions of eleven elements in Florida soils. J. Environ. Qual., 26:769-775, 1997.

MALAVOLTA, E. Micronutrientes e metais pesados: mitos, mistificação e fatos. São Paulo, Produquimica, 1994. 140p.

MENDONÇA, E.S. \& MATOS, E.S. Matéria orgânica do solo: Métodos de análise. Viçosa, MG, Universidade Federal de Viçosa, 2005. 107p.

MINERAIS DO PARANÁ S.A. - MINEROPAR. Geoquímica de solo - Horizonte B. In: Levantamento Geoquímico Multielementar do Estado do Paraná. Curitiba, PR, Mineropar, 2005. v.2p. 407p.

MOURA, C.V.A. Aplicação de tratamento estatístico multivariante em dados geoquímicos de solo no mapeamento geológico na província de Carajás. R. Bras. Geoci., 15:241-248, 1985.

NAIDU, R.; BOLAN, N.S.; KOOKANA, R.S. \& TILLER, K.G. Ionic-strength and $\mathrm{pH}$ effects on the sorption of cadmium and the surface charge of soils. Eur. J. Soil Sci., 45:419429, 1994.

OLIVEIRA, T.S.; COSTA, L.M. \& CRUZ, C.D. Importância relativa dos metais pesados do solo na identificação e separação de materiais de origem. R. Ceres, 45:359-371, 1998.

OLIVEIRA, F.C. \& MATIAZZO, M.E. Mobilidade de metais pesados em Latossolo Amarelo distrófico tratado com lodo de esgoto e cultivado com cana-de-açúcar . Sci. Agríc., 58:807-812, 2001

PARDO, M.T. Sorption of lead, copper, zinc, and cadmium by soils: Effect of nitriloacetic acid on metal retention. Comm. Soil Sci. Plant, 31:31-40, 2000.

ROSS, S.M. Toxic metals in soil-plant systems. Chichester, John Willey \& Sons, 1994. 469p.

SINGH, B.R. \& STEINNES, E. Soil and water contamination by heavy metals. In: LAI, R. \& STEWART, B.A, eds. Advances in soil science: Soil process and water quality. Ankeny, Lewis, 1994. p.233-237.

SOARES, M.R. Coeficiente de distribuição (Kd) de metais pesados em solos do Estado de São Paulo. Piracicaba, Escola Superior de Agricultura Luiz de Queiroz, 2004. 202p. (Tese de Doutorado)

SU, Y. \& YANG, R. Background concentrations of elements in surface soils and their changes as affected by agriculture use in the desert-oasis ecotone in the middle of Heihe River Basin, North-west China. J. Geochem. Explor., 98:57-64, 2008.

TACK, F.M.G.; VERLOO, M.G.; VANMECHELEN, L. \& van RANST, E. Baseline concentrations levels of trace elements as a function of clay and organic carbon contents in soils in Flanders (Belgium). Sci. Total Environ., 201:113-123, 1997.

UNITED STATES ENVIRONMENTAL PROTECTION AGENCY - USEPA. Soil screening guidance: Thecnical background document. Washington, Office of Solid Waste and Emergency Response, 1996. 168p. 
UNITED STATES ENVIRONMENTAL PROTECTION AGENCY - USEPA. Batch-type Procedures for Estimating Soil Adsorption of Chemicals. Washington, Technical Resource Document, EPA/530-SW-87-006-F, 1992. 99p.
UNITED STATES ENVIRONMENTAL PROTECTION AGENCY - USEPA. Microwave assisted acid digestion of sediments, sludges, soils and oils. (Technical Resource Document, EPA SW-846/3052). Disponível em: <http:// www.epa.gov/epaoswer/ hazwaste/test/pdfs/3052.pdf>. Acesso em 19 fev. 2007. 